\title{
Investigating Dermatologic Side Effects of Anti-Cancer Chemotherapeutic Agents
}

\author{
(1) Melis Gönülal', (1) Murat Keser², (1) Aylin Öztürk \\ ${ }^{1}$ Izmir Tepecik Training and Research Hospital, Clinic of Dermatology, Izmir, Turkey \\ 2Izmir Tepecik Training and Research Hospital, Clinic of Oncology, Izmir, Turkey
}

\section{ABSTRACT}

Background: Various chemotherapeutic agents are used in cancer treatment. The side effects they create on skin are sometimes the same and sometimes different. We purposed to investigate skin, skin appendages and mucosa adverse effects of anti-cancer chemotherapeutic agents with literature in our study.

Materials and Methods: Age, gender, type of cancer, used anti-cancer chemotherapeutic agents, number of cycles of patients were noted. Skin, skin appendages and mucosa adverse effects of anti-cancer chemotherapeutic agents were recorded.

Results: Forty-eight patients were included in our study, 12 of them (25\%) were male and 36 of them (75\%) were female. We observed different skin, skin appendages and mucosa adverse effects of anti-cancer chemotherapeutic agents and presented them in tables.

Conclusion: Chemotherapeutics can have beneficial effects as well as damaging effects on various organs. We compared dermatologic side effects of chemotherapeutic agents in the light of literature in our study. We also mentioned dermatologic side effects that haven't been mentioned in the literature yet. We believe that our research will contribute to the literature with all these aspects.

Keywords: Anti-cancer chemotherapeutic agent, Skin, Skin appendage, Mucosa, Dermatologic side effects

\section{Introduction}

Cancer is a complex disease that occurs with the uncontrolled division and proliferation of cells and consists of genetic and environmental conditions. There are various treatment methods for cancer, one of them is chemotherapy. The main purpose of chemotherapy is to kill cancer cells using chemotherapeutic agents and cytotoxic anti-neoplastic agents play the leading role in this type of therapy [1]. Conventional anticancer treatments target cells that can divide rapidly. Their side effects are observed more common in tissues containing proliferative cells, in addition, their systemic side effects are more prominent than their side effects on skin. The frequency of skin side effects of the targeted anticancer treatments is gradually increasing and their skin side effects are more prominent than their systemic side effects [2,3]. In our study, we aimed to evaluate the skin side effects of anticancer drugs in the light of the current literature.

\section{Materials and Methods}

This study was designed as a prospective single-center study, and it was conducted under the ethical principles reported in the Declaration of Helsinki. It was approved by the University of Health Sciences Turkey, Izmir Tepecik Training and Research Hospital Ethical Review Committee. Patients aged more than 18 who presented to the Oncology Outpatient Clinic of Izmir Tepecik Training and Research Hospital between September 2019 and March 2020 recruited into this study. Both verbal and written informed consents were 
obtained from study participants. The patients included in the study were those who were currently receiving chemotherapy for their own diseases. The patients who were followed up in the oncology clinic of our hospital and had side effects on the skin, mucosa and skin appendages because of their chemotherapy, were included for the research. We noted the age, gender, cancer type, chemotherapy drug, number of cycles, examination findings about side effects of chemotherapy on skin-mucosa-skin appendages of the patients. Dermatological regions with side effects were examined under six sub-units in terms of localizations: hair, scalp, nail, oral mucosa, genital mucosa, conjunctiva, skin.

\section{Statistical Analysis}

Statistical analyzes were performed using Statistical Package for Social Sciences (SPSS v.17.0, IBM Corporation, Armonk, NY, US) software. The results were evaluated with descriptive analysis.

\section{Results}

During the study period, 48 patients were included to our study (12 male, 25\%; 36 female, 75\%). The mean age of all patients was 55.46 \pm 12.5 (minimum: 23, maximum: 82). We determined the mean age of female patients as $54.53 \pm 12.56$ (minimum: 37 , maximum: 82 ) and of male patients as $58.25 \pm 12.51$ (minimum: 23 , maximum: 75). Cancer types of the patients, types of chemotherapy and the side effects of these chemotherapies are shown in the tables (Table 1, 2).

\section{Discussion}

Various chemotherapeutic agents have been used for cancer treatment over the past 20 years. These drugs are divided into some

\begin{tabular}{|l|l|l|}
\hline Tablo 1. Cancer types of patients & Percent (\%) \\
\hline Types of cancer & Number (gender) & 2.1 \\
\hline Lung & 1 (male) & 2.1 \\
\hline Brain & 1 (male) & 2.1 \\
\hline Kidney & 1 (male) & 2.1 \\
\hline $\begin{array}{l}\text { Endometrium } \\
\text { leiomyosarcoma }\end{array}$ & 1 (female) & 2.1 \\
\hline Liver & 1 (male) & 8.3 \\
\hline Colon & 4 (3 female, 1 male) & 4.2 \\
\hline Larynx & 2 (male) & 58.3 \\
\hline Breast & 28 (female) & 2.1 \\
\hline Stomach & 1 (female) & 2.1 \\
\hline Osteosarcoma & 1 (female) & 4.2 \\
\hline Ovary & 2 (female) & 2.1 \\
\hline Prostate & 1 (male) & 4.2 \\
\hline Rectum & 2 (male) & 4.2 \\
\hline Testis & 2 (male) & \\
\hline & & \\
\hline
\end{tabular}

subgroups according to their mechanisms; signal transduction inhibitors [epidermal growth factor receptor (EBFR) antagonists, multikinase inhibitors], proteasome inhibitors, spindle inhibitors (taxanes, vinca alkaloids), antimetabolites (purine analogs, primidine analogs), genotoxic agents. These chemotherapestics may have various dermatological side effects [4].

In our study, it is thought that the papulopustular rash is mostly due to cetuximab if we compare the dual use of 5-fluoroucil (5FU) and cetuximab with the triple use of 5-FU, oxaliplatin and cetuximab. Oxaliplatin didn't cause any additional skin findings in these two drugs combinations. Cetuximab had caused only herpes zoster in its single use. The most common side effect of cetuximab, an EBFR inhibitor, is papulopustular rash seen in $60 \%$ to $80 \%$ of patients [5]. According to the data of our study, this side effect can be brought to the fore even in combination therapies. In the work of Yazganoğlu and Baykal [6], papulopustular rash on the face of a female patient 62-year-old, had been observed in the dual use of 5-FU and cetuximab, too. Interestingly, the triple use of 5-FU, cetuximab and carboplatin caused seborrheic dermatitis and xerosis as dermatological side effects. Platins are in the cytotoxic cancer treatment category and their most common side effect is alopecia [7]. In the combination therapies using oxaliplatin and carboplatin, alopecia didn't occur. We thought that other agents in the combination could prevent the occurrence of alopecia. In the triple use of 5-FU, irinotecan, cetuximab, oral mucosa pigmentaiton and eczema on the hands were observed, these are rare side effects. We determined oral aphthaes and desquamation on hands and feet in the triple use of 5-FU, irinotecan and aflibersept in our study, papulopustular rash didn't occur. Aflibersept has inhibitory effects on vascular endothelial growth factor (VEGF), can lead to thrombotic events [8]. We thought the cause of oral aphthae of our case was aflibersept. In addition, there were publications in the literature that aflibersept caused ulcers in the abdominal wound scar and around the stoma $[8,9]$. According to tha data of our study, carboplatin and oxaliplatin could occur side effects more quickly. It could be observed that 5-FU, cetuximab, irinotecan and aflibersept were slow to cause dermatologic side effects.

Bevacizumab is a VEGF inhibitor and papulopustular rash because of it has been reported in a small number of cases [6]. It is known that irinotecan causes papulopustular rash. In our research, in the dual use of bevacizumab and irinotecan, the occurence of telangiectasia contradicts the mechanism of bevacizumab, this suggests that irinotecan may be more dominant in the formation of telangiectasia. Additional studies are needed in this topic. We observed that dominant side effect was erythema on face and neck in the triple use of capecitabine, oxaliplatin, bevacizumab. Capecitabine is the prodrug of 5-FU. We thought that capecitabine was responsible for this erythema because the 
dominant dermatologic side effect of oxaliplatin was alopecia and of bevacizumab was ulcer on skin. Photosensitive lichenoid skin reaction had been reported in a female patient 73-year-old because of capecitabine in the literature [10]. According to our study, side effects of paclitaxel were observed until the $12^{\text {th }}$ cycle and in the dual use of paclitaxel and carboplatin dermatologic side effects occured earlier and less amount. Dominant side effects of taxans (dosetaxel, paclitaxel) are reversible alopecia, dorsal hand foot syndrome, edema, flexural and intertriginous rash, onycholysis, mucositis. It was reported that hand foot syndrome was caused

Tablo 2. Chemotherapies and side effects on skin-mucosa-skin appendages

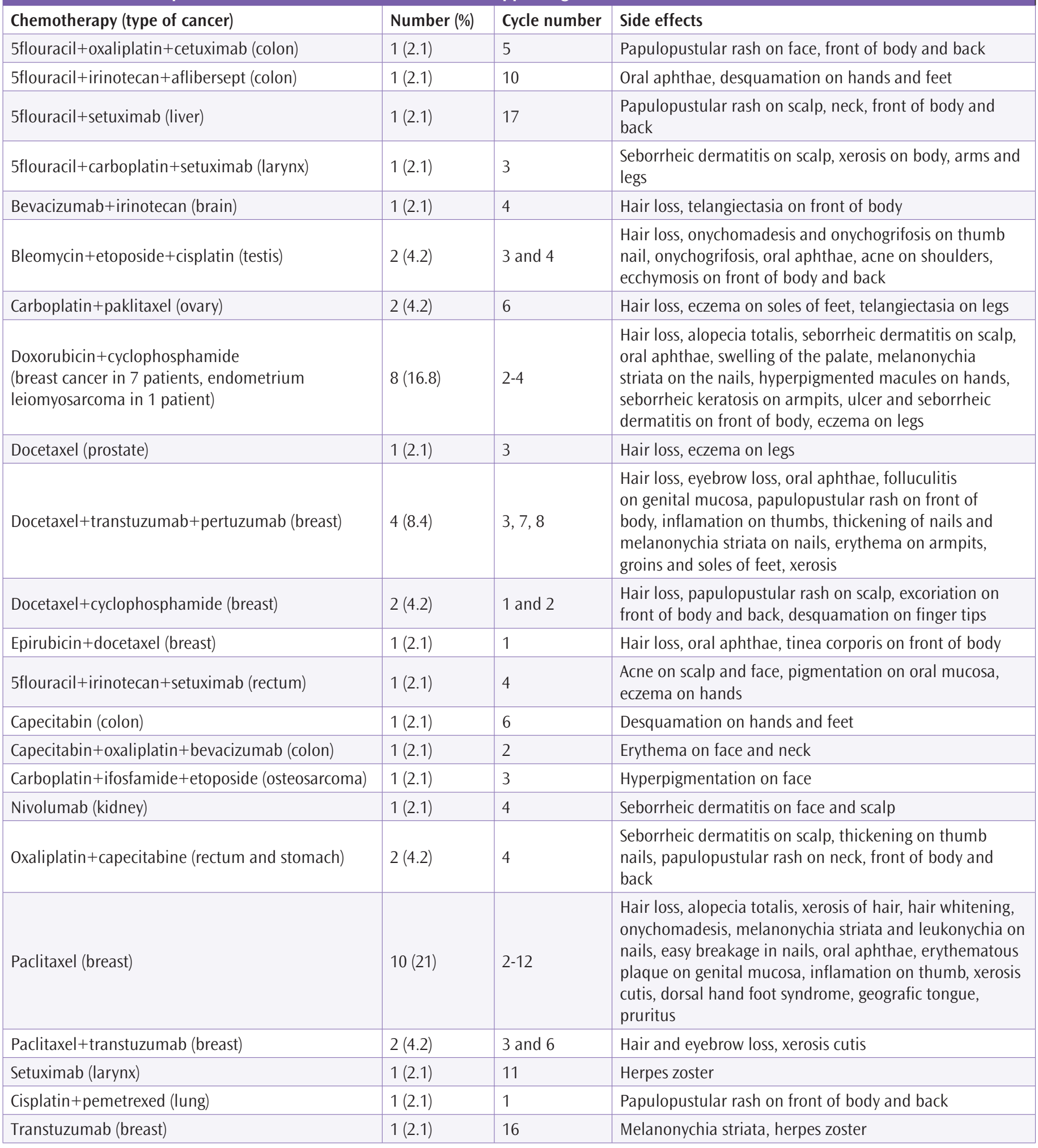


by especially docetaxel [11]. In our research, we observed that this syndrome could be caused by paclitaxel although rare. These dominant side effects were in line with the results of our study. The side effects of transtuzumab are mild on the skin. In the study of Adachi et al. [12], skin side effects in 25 patients, nail side effects in 14 patients and both skin and nail side effects in 12 patients were observed. Eruptions on face and body, thinning of skin of hands and feet, pruritus, xerosis cutis, softening and thinning of nails, paronychia, discoloration were determined. In our study, in the single use of transtuzumab, melanonychia striata and herpes zoster occured. To sum up, we thought that carboplatin and transtuzumab could decrease the side effects of paclitaxel in the combination treatments.

The most side effects of capecitabine are edema on hands and feet, feeling of tightness and pain [13]. In our study we observed desquamation on hands and feet, probably, we thought that there was edema firstly and desquamation developed as the skin healed. In the research of Prussick et al. [14], in the triple use of carboplatin, ifosfamide and etoposide, periorbital erythema, mucositis, erythema on face and groin occured in eight patients. In addition there are publications that reported hyperpigmentation because of ifosfamide in the literature [15]. These results were consistent with our study.

In the study of Swain et al. [16], alopecia, rash on body, pruritus and xerosis cutis occured in the triple use of pertezumab, transtuzumab and dosetaxel. Baselga et al. [17] detemined alopecia, rash on body, peripheral edema, xerosis cutis in their research. In the research of Nakatsukasa et al. [18], peripheral edema and rash on body occured in the dual use of cyclophosphamide and dosetaxel in 48 female patients. Nitz et al. [19] determined hand and foot syndrome and mucositis/stomatitis in 198 patients. These all results were consistent with our research.

In the study of Petrioli et al. [20] stomatitis was observed in 10 patients of those using epirubicin only, in five patients of those using dosetaxel only. Hand and foot syndrome was determined in six cases of those using epirubicin only and in three cases of those using dosetaxel only. Accoding to these results, epirubicin has a higher potential to cause side effects on skin and its appendages than docetaxel. We observed in our study that hair loss, oral aphthae, tinea corporis on front of body could occur in the dual use of epirubicin and dosetaxel.

Nivolumab is a chemotherapeutic agent from the programmed cell death inhibitor 1 group. It causes lichenoid reactions, eczema, vitiligo and pruritus especially [21]. Incompatible with this study we observed that nivolumab could cause seborrheic dermatitis on face and scalp.

\section{Study Limitations}

Our study has some limitations which need to be considered while evaluating its findings. First, it is a single-center study. Second, although oncologist was always working in the same part of hospital, dermatologists were working in the three different parts of the hospital during the process of research, therefore patients who had skin damage because of anticancer drugs, couldn't be sometimes examined by dermatologists. If dermatologists and oncologist always worked in the same part of hospital, the number of patients would be higher.

\section{Conclusion}

In conclusion, chemotherapeutics can have beneficial effects as well as damaging effects on various organs. We compared dermatologic side effects of chemotherapeutic agents in the light of literature in our study. We also mentioned dermatologic side effects that haven't been mentioned in the literature yet. We believe that our research will contribute to the literature with all these aspects.

\section{Ethics}

Ethics Committee Approval: It was approved by the University of Health Sciences Turkey, Izmir Tepecik Training and Research Hospital Ethical Review Committee (protocol number: 2019/13-30, date: 11/09/2019).

Informed Consent: Both verbal and written informed consents were obtained from study participants.

Peer-review: Externally and internally peer-reviewed.

\section{Authorship Contributions}

Surgical and Medical Practices: M.G., M.K., A.Ö., Concept: M.G., M.K., A.Ö., Design: M.G., M.K., A.Ö., Data Collection or Processing: M.G., M.K., A.Ö., Analysis or Interpretation: M.G., M.K., A.Ö., Literature Search: M.G., M.K., A.Ö., Writing: M.G., M.K., A.Ö.

Conflict of Interest: No conflict of interest was declared by the authors.

Financial Disclosure: The authors declared that this study received no financial support.

\section{References}

1. Baykara 0. Current modalities in treatment of cancer. Balikesir Saglik Bil Derg 2016;5:154-165

2. Kaul S, Kaffenberger BH, Choi JN, Kwatra SG. Cutaneous adverse reactions of anticancer agents. Dermatol Clin 2019;37:555-568.

3. Shi VJ, Levy LL, Choi JN. Cutaneous manifestations of nontargeted and targeted chemotherapies. Semin Oncol 2016;43:419-425.

4. Fabbrocini G, Cameli N, Romano MC, Maria Mariano, Panariello L, Bianca D, Monfrecola, G. Chemotherapy and skin reactions. J Exp Clin Cancer Res 2012;31:50. 
5. Pinto C, Barone CA, Girolomoni G, Russi EG, Merlano MC, Ferrari D, Maiello E. Management of skin toxicity associated with cetuximab treatment in combination with chemotherapy or radiotherapy. Oncologist 2011;16:228238.

6. Yazganoğlu KD, Baykal C. Acneiform eruption and other dermatologic side effects induced by targeted cancer therapy: a retrospective analysis. Türkderm 2012;46:84-89.

7. Özgül N, Erten Ö, Düzgüner S, Taner Turan T, Kög İ, Boran N, Balta İ, Köse MF. Paclitaxel/Platinum combination regimen and alopecia. J Turk Soc Obstet Gynecol 2012;9:94-98.

8. Rivas-Tolosa N, Calomarde L, Bancalari B, Guillén C, Sanmartín O. Ulcerations on abdominal wound scar associated with aflibercept therapy. J Dermatol 2016;43:1095-1096.

9. Fujiwara S, Chida Y. Skin ulceration around stoma associated with aflibercept. BMJ Case Rep 2019;12:e232278.

10. Shah RA, Bennett DD, Burkard ME. Photosensitive lichenoid skin reaction to capecitabine. BMC Cancer 2017;17:866.

11. Sibaud V, Lebœuf NR, Roche H, Belum VR, Gladieff L, Deslandres M, Montastruc M, Eche A, Vigarios E, Dalenc F, Lacouture ME. Dermatological adverse events with taxane chemotherapy. Eur J Dermatol 2016;26:427-443.

12. Adachi S, Yoshimura T, Matsuoka T, Okada K, Yasuda T, Kamei K. Appearance of skin and nail toxicity in patients with breast cancer who underwent trastuzumab-containing chemotherapy. Gan To Kagaku Ryoho 2011;38:14531456.

13. Park JY. Analysis of data on capecitabine-related adverse drug reactions from the Korean adverse event reporting system database. Eur J Oncol Nurs 2018;34:55-60.

14. Prussick R, Horn TD, Wilson WH, Turner MC. A Characteristic eruption associated with ifosfamide, carboplatin, and etoposide chemotherapy after pretreatment with recombinant interleukin-1 alpha. J Am Acad Dermatol 1996;35:705-709.

15. Teresi ME, Murry DJ, Cornelius AS. Ifosfamide-induced hyperpigmentation. Cancer 1993;71:2873-2875.

16. Swain SM, Baselga J, Kim SB, Ro J, Semiglazov V, Campone M, Ciruelos E, Ferrero JM, Schneeweiss A, Heeson S, Clark E, Ross G, Benyunes MC, Cortés J, Cleopatra Study Group. Pertuzumab, trastuzumab, and docetaxel in HER2positive metastatic breast cancer. N Engl J Med 2015;372:724-734.

17. Baselga J, Cortés J, Kim SB, Im SA, Hegg R, Im YH, Roman L, Pedrini JL, Pienkowski T, Knott A, Clark E, Benyunes MC, Ross G, Swain SM, Cleopatra Study Group. Pertuzumab plus trastuzumab plus docetaxel for metastatic breast cancer. N Engl J Med 2012;366:109-119.

18. Nakatsukasa K, Koyama H, Oouchi Y, Imanishi S, Mizuta N, Sakaguchi K, Fujita Y, Fujiwara I, Kotani T, Matsuda T, Fukuda K, Morita M, Kawakami S, Kadotani Y, Eiichi Konishi, Akio Yanagisawa, Tetsuya Taguchi. Docetaxel and cyclophosphamide as neoadjuvant chemotherapy in HER2-negative primary breast cancer. Breast Cancer 2017;24:63-68.

19. Nitz U, Gluz O, Clemens M, Malter W, Reimer T, Nuding B, Aktas B, Stefek A, Pollmanns A, Lorenz-Salehi F, Uleer C, Krabisch P, Kuemmel S, Liedtke C, Shak S, Wuerstlein R, Christgen M, Kates RE, Kreipe HH, Harbeck N, West German Study Group PlanB Investigators. West German study plan B trial: adjuvant four cycles of epirubicin and cyclophosphamide plus docetaxel versus six cycles of docetaxel and cyclophosphamide in HER2-negative early breast cancer. J Clin Oncol 2019;37:799-808.

20. Petrioli R, Roviello G, Zanotti L, Roviello F, Polom K, Bottini A, Marano L, Francini E, Marrelli D, Generali D. Epirubicin-based compared with docetaxelbased chemotherapy for advanced gastric carcinoma: a systematic review and meta-analysis. Crit Rev Oncol Hematol 2016;102:82-88.

21. Collins LK, Chapman MS, Carter JB, Samie FH. Cutaneous adverse effects of the immune checkpoint inhibitors. Curr Probl Cancer 2017;41:125-128. 